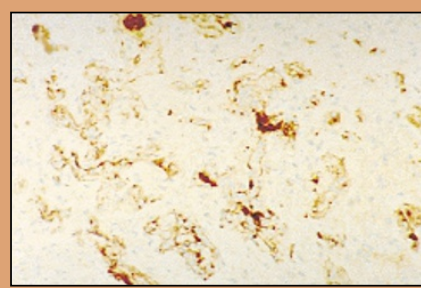

In the search for anticancer drugs, a promising area involves the design of smallmolecule inhibitors of growth factors active in malignancies. PDGF and its receptors are implicated in tumorigenesis and angiogenesis, prompting Sebti and colleagues to design a synthetic molecule, called GFB-111, that binds to PDGF. Their new molecule has four-fold symmetry consisting of four identical peptide loops with negative and hydrophobic residues tailored to complement the specific PDGF surface involved in binding to its receptor, thereby preventing binding. Human tumor-bearing nude mice injected with GFB-111 had significant reduction of both tumor growth and angiogenesis (see p. 1065).

\section{Protein identification at nanomolar concentration}

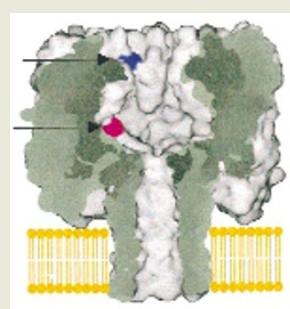

Stochastic sensing of single molecules passing through ion channels is becoming an established technology for detecting minute quantities of analytes in solution; however, until now this approach has been limited to detection of molecules small enough to pass through the channel. In this issue, Bayley and colleagues present a new type of biosensor that can detect even macromolecules in nanomolar concentrations. Within the pore of an $\alpha$-hemolysin channel, they tethered a polyethylene glycol chain with a biotinyl group attached to its free end. When the modified channel was incorporated into a lipid bilayer, the biotinyl group was free to move from one side of the membrane to the other, where it could be captured by streptavidin. Capture was detected by changes in ionic current through the channel. The method can potentially provide both quantification and identification of a protein analyte at the single-molecule level (see pp. 1037 and 1091).

\section{$J J$}

This month in Nature Biotechnology written by Natalie DeWitt, Judy Jamison, Andrew Marhsall, and Meeghan Sinclair.

\section{A FACS screen for improving enzymes}

For creating improved enzymes by directed evolution, it is thought that the use of larger libraries will yield variants with the most desirable properties. However, rapidly sifting through tens of thousands of variants to find that special enzyme can create a bottleneck. In this issue, Olsen et al. use FACS to rapidly sort through a large library of OmpT protease variants displayed on the surface of bacteria. This allowed them access to the substrate- a peptide containing a nonpreferred OmpT cleavage site attached to fluorescent FRET partners. Cleavage disrupts FRET quenching, yielding a fluorescent signal that corresponds to the activity of the protease variant. Using the method, they screened a large library of $\sim 6 \times 10^{5}$ random OmpT variants and found ones with 60 -fold higher activities for the nonpreferred cleavage site. The method can potentially be applied to a variety of proteins displayed on the surface of bacteria or yeast (see p. 1071).

$N D$

\section{Synthetic transcriptional activators}

Transcriptional activators are currently thought to consist of 40-80 amino acid tracts that promote target gene expression through multiple contacts with proteins in the transcriptional apparatus. However, little is known about the sequence and structural requirements of minimal mammalian activation domains. On page 1080, Frangioni et al. describe the results of a phage display screen of a library of eight-amino acid peptides for binding to the KIX domain of the transcriptional co-activators p300 and CBP. The peptides identified were capable of mediating transcriptional coactivation when fused to a DNA-binding domain. The results suggest that these minimal activation domains might be engineered to create precise activators for transcription factor-mediated gene therapy.

MS

\title{
Review
}

Although considerable attention has been paid to the use of microbial genomics in medicine, genomic information also promises to make an important contribution to a diverse range of other environmental, food, and industrial applications of biotechnology. As gene sequencing technology becomes more routine, researchers are determining the sequences of more obscure microorganisms and delving into the diversity of the microbial world with the aim of discovering new products. It is hoped that genome data on nonpathogenic bacteria will lead to the discovery of biocatalysts resistant to extremes of $\mathrm{pH}$, temperature, or solvents; nutritionally beneficial bacteria for probiotics; new types of streptomycete antibiotics; and microorganisms with enhanced capabilities to degrade xenobiotic compounds at contaminated sites or leach minerals in biomining. In this issue, Nelson et al. (see p. 1049) summarize recent progress in microbial genome projects, describe the challenges in sequencing a nonpathogenic microorganism, and briefly discuss the potential of complete genome sequence information for providing insights into ways of engineering new and improved activities.

\section{Technical Reports}

On page 1105, Nienaber et al. describe a structure-based drug discovery approach that employs a new crystallographic screening method, using the anticancer target urokinase as a model. Urokinase crystals were exposed to mixtures of compounds, and ligands monitored by electron density maps that reveal the bound and unbound forms of the crystal. Using the approach, those ligands that bind away from a target site can be eliminated, and details of the ligand-target interaction from the X-ray data can be used as leads for structure-directed optimizations.

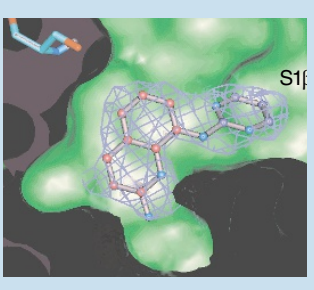

Flanagan and colleagues report a simplified method for in vivo footprinting that can provide extremely rapid and sensitive analysis of transcription binding and chromatin structure of promoters. Their protocol yielded longer reads and allowed a statistical study of band variation (see p. 1108). 